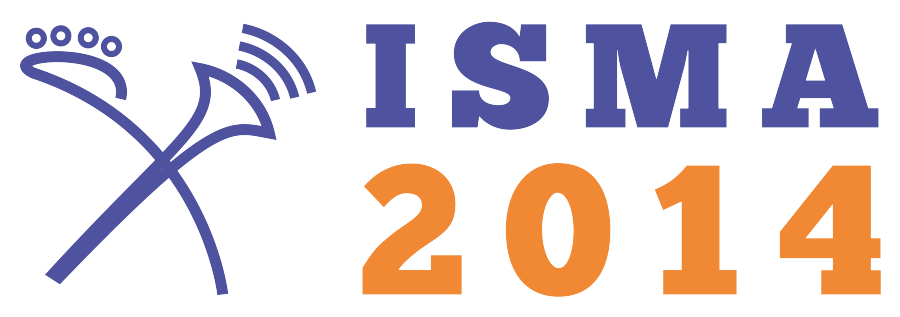

\title{
Sound Radiation Characteristic of a Shakuhachi with different Playing Techniques
}

\section{T. Ziemer}

University of Hamburg, Neue Rabenstr. 13, 20354 Hamburg, Germany tim.ziemer@uni-hamburg.de 
The shakuhachi, a Japanese flute, is a rather small instrument with a simple geometry. Still, it appears to have a complicated spatial sound radiation characteristic. This effect results from interference of sound emanating from finger holes and the blowing hole as well as diffraction around and acoustic shadow behind the instrumentalist. Even in absence of room reflections, the pure direct sound of musical instruments already creates the impression of a certain extent of the source. This perceived extent is especially large for listeners close to the instrument and decreases with distance. This effect is investigated in more detail on the shakuhachi.

The sound of a shakuhachi is recorded in an anechoic chamber by a circular microphone array consisting of 128 microphones. Amplitude and phase per frequency and angle around the instrument are measured. Interaural phaseand amplitude differences as well as the correlation of the signals arriving at the two ears are calculated for several listening positions at various angles and distances. These parameters are compared between different playing techniques. It is discussed how far the parameters are suitable to explain the perception of the spatial source extent.

\section{Introduction}

It is known that in a free field sound sources appear smaller with increasing distance because their wave fronts more and more resemble a monopole and thus interaural differences diminish. In this paper considerations are made which parameters affect the perception of source extent. These parameters need to show the same behavior, i.e. decrease with distance. A method is proposed to calculate sound field quantities at different positions in space from circular microphone array recordings by propagating the sound field, considering the musical instrument as complex point source. This simplification is physically untrue but enables us to gain interaural differences for numerous listening positions. Thus, radiation characteristics of musical instruments can be analyzed and compared which is exemplarily done with two playing techniques of the shakuhachi, namely a simply blown and an overblown note. The results confirm the applicability of this method and suggest, together with results from a simple listening test, which physical parameters could be responsible for the perception of source extent and its decrease with increasing distance.

\section{Sound radiation of instruments}

Meyer extensively investigated the sound radiation characteristics of musical instruments by far field recordings with circular microphone arrays [1]. He found out that the complicated radiation patterns of flutes are due to superposition of sounds radiating especially from the blow hole and the first open tone hole, which acts as open end of the tube. Furthermore, he observed a wave shadowing behind the flutist's head at high frequencies. Later, e.g. Pätynen and Lokki used layers of circular microphone arrays to measure radiation patterns of symphony orchestra instruments in three dimensions [2]. Zotter et al. used spherical microphone arrays to measure the three-dimensional radiation characteristics of musical instruments at discrete positions in space and combine it with spherical harmonic decomposition to predict sound field quantities in between the actually recorded positions [3]. They used 22 and 26 microphones to capture the complete three-dimensional radiation characteristics. In this paper, only the horizontal plane is investigated with 128 microphones to gain enough information to reliably predict sound field quantities around the instruments at different distances.

\subsection{Sound radiation of the shakuhachi}

According to Meyer the flute mainly radiates sounds from the blow hole and the first open tone hole. The phase relation between those two sound-radiating areas depends on the number of half wavelength between the holes. For odd numbers, the ends radiate in phase, for even numbers out of phase. This roughly results in monopole and dipole characteristics. Bader et al. found the phase relation to be more complicated in case of the shakuhachi [4]. Furthermore, the sound pressure at the labium decreases with higher partials while the pressure radiation from the finger hole wanders down and splits into two regions. This explains how the diverse radiation patterns of the shakuhachi occur.

\section{Experimental setup}

128 omnidirectional electret microphones are arranged in a free field room on a circle with a radius of $1 \mathrm{~m}$. So, adjacent microphones have a distance of about $0.05 \mathrm{~m}$, providing one microphone every $2.8^{\circ}$. An instrumentalist is in the center of the array facing the first microphone. Only the horizontal plane in height of the instrumentalists head is considered. Figure 1 is a photo of the investigated shakuhachi. The note re, which is generated by covering the first three finger holes, is played with two different techniques, once blown and once overblown. The simply blown re should result in the note $g^{1}$, the overblown re in its octave $g^{2}$. The player tried to generate a pure harmonic sound with little noise, articulation, amplitude- or frequency modulations. All microphones simultaneously perform a two-second recording of the quasi-stationary part of each played note with a sample rate of $48 \mathrm{kHz}$ and a sample depth of 24Bit. The $2 \times 128$ recordings are analyzed. From these signals, interaural differences for listeners at different locations around the instrument are calculated using the introduced complex point source model and compared with subjective judgments of source size and distance as gained from a simple listening test.

Figure 1: Photo of the investigated shakuhachi. 


\subsection{The complex point source model}

It is feasible to consider a musical instrument a point source if its volume is small compared to the radiated wave length. This is of course a simplification which is physically untrue. Naturally, musical instruments radiate sounds from different parts of the body and the enclosed air. The radiated sounds interfere and create different amplitudes and phases at the different locations in space. Thus, there is no exclusive source point. Therefore, the head of the musician is taken as source point for the investigation, as commonly done, e.g. in [2]. This simplification means that all sound is considered as originating fom one common source point, namely the center of the microphone array, at which a complex point source radiates sound. In the frequency domain, this situation is described by the twodimensional inhomogeneous Helmholtz equation which can be formulated as a set of ordinary differential equations in spherical coordinates:

$$
\begin{array}{r}
\frac{\mathrm{d}^{2} \Gamma(\omega, \varphi)}{\mathrm{d} \varphi^{2}}+m^{2} \Gamma(\omega, \varphi)=P_{s}(\omega, \varphi) \\
\frac{1}{r^{2}} \frac{\mathrm{d}\left(r^{2} \frac{\mathrm{d} G(\omega, r)}{\mathrm{d} r}\right)}{\mathrm{d} r}+k^{2} G(\omega, r)-\frac{n(n+1)}{r^{2}} G(\omega, r)
\end{array}
$$

Here,

$$
\mathbf{r}=\left[\begin{array}{l}
r \\
\varphi
\end{array}\right]
$$

is the position vector containing distance $r$ from the origin $\mathbf{r}_{0}$ and azimuth angle $\varphi . \quad P_{s}\left(\omega, \mathbf{r}_{0}\right)=P_{s}(\omega, \varphi) P_{s}\left(\omega, r_{0}\right)$ is the yet unknown source spectrum, a solution to the homogeneous Helmholtz equation. Solutions to the first differential equation of Eq. (1) are the radiation characteristic $\Gamma(\omega, \phi)$ of the musical instruments in the horizontal plane. These are implicitly measured for 128 angles by the microphone array. Solutions to the second differential equation are complex transfer functions. One solution is the free field Green's function

$$
G(\omega, r)=\frac{1}{r} e^{-\imath k r}
$$

with Euler's number $e$, imaginary unit $\imath=\sqrt{-1}$, the wave number $k=\frac{\omega}{c}$ where $c$ is the speed of sound. From the relationship

$$
P(\omega, \mathbf{m})=P_{s}(\omega, 0) \Gamma(\omega, \varphi) G(\omega, r)
$$

the sound field measured at the microphone angles can be propagated back and forth towards or away from the source. Basically, the spectra recorded at the microphone positions $P\left(\omega, \mathbf{r}_{m}\right)$ are nothing but the radiation characteristic of the source $\Gamma(\omega, \varphi)$, reduced in amplitude and shifted in phase according to the complex transfer function $G(\omega, r)$. The complex point source model is only valid in the far field which is defined as $r \gg \frac{\lambda}{2 \pi}$ where $\lambda=\frac{c}{f}$ is the wavelength. Considering " $\gg$ " as one order of magnitude the microphone radius of $1 \mathrm{~m}$ lies in the far field for frequencies above roughly $546 \mathrm{~Hz}$. However, musical instruments show a monopole-like radiation in that frequency region anyway, at $1 \mathrm{~m}$ as well as in he far field. So the method gives reasonable spatial results and only leads to an overemphasis of low frequencies. The idea of a complex point source is similar to the single point multipole method which is described e.g. in [5]. But in this case the radiation is neither decomposed to point sources of high order or to spherical harmonics-as in $[3,5]$-nor interpolated to receive sound field quantities at positions in between the discrete recording angles. Only the actual recording signals are taken and forward-propagated to calculate interaural signal differences for listeners situated at different angles and distances.

\subsection{Interaural measures}

Investigated physical parameters are the interaural level difference $I L D$ and interaural phase difference $I P D$ of partials, the binaural quality index $B Q I$ of the bandpassed time series and their fluctuations around the source quantified by the range $R$ and standard deviation $\sigma$ from the arithmetic mean $\oslash$.

A discrete Fourier transform DFT of one second of quasi-stationary sound transforms the measured time series $p(t, \mathbf{r})$ into frequency domain $P(\omega, \mathbf{r})$. In frequency domain $I L D$ and $I P D$ can simply be calculated for spectral components:

$$
\begin{aligned}
& I L D=\hat{A}\left(\omega, \varphi_{L}\right)-\hat{A}\left(\omega, \varphi_{R}\right) \\
& I P D=\phi\left(\omega, \varphi_{L}\right)-\phi\left(\omega, \varphi_{R}\right)
\end{aligned}
$$

The amplitude $\hat{A}$ is the absolute value of the spectral component $P(\omega, \mathbf{r})$ and phase $\phi$ its argument, accordingly. This is valid since the sounds are quasi-stationary. The subscripts $L$ and $R$ denote "left" and "right" ear. The $I L D$ is fairly known to have an impact on the perceived source position. Different $I L D$ for different spectral components may thus create the impression of an increased apparent source width ASW. IPD are known to have a similar effect as $I L D$ and thus additionally contribute to a perception of width. Griesinger found that fluctuations of interaural intensity differences and interaural time delays affect the perception of spaciousness [6]. Thus, the fluctuations are quantified in this investigation by means of $R$ and $\sigma$.

$B Q I$ is a parameter which is utilized in room acoustical investigations, see [7]. It is derived from the interaural cross correlation coefficient IACC as calculated from dummy head recordings:

$$
\begin{aligned}
I A C F_{0,80 \mathrm{~ms}} & =\frac{\int_{0}^{80 \mathrm{~ms}} p_{L}(t) p_{R}(t+\tau) \mathrm{d} t}{\sqrt{\int_{0}^{80 \mathrm{~ms}} p_{L}^{2}(t) \mathrm{d} t \int_{0}^{80 \mathrm{~ms}} p_{R}^{2}(t) \mathrm{d} t}} \\
I A C C & =\max |I A C F(\tau)| \\
B Q I & =1-I A C C
\end{aligned}
$$

The interaural cross correlation function $I A C F$ is a cross correlation of bandlimited temporal signals which contain three octave bands around the center frequencies $500 \mathrm{~Hz}$, $1 \mathrm{kHz}$ and $2 \mathrm{kHz}$. In room acoustics, $\tau$ is a value to shift one signal up to $\pm 1 \mathrm{~ms}$ to compensate for the dependency of the $I A C C$ on the azimuth position of the source. Lateral signals reach the ears with an interaural time difference of up to almost $1 \mathrm{~ms}$ which affects the correlation even though the perceived source width is almost independent of incidence angle. This is compensated by shifting one signal and taking the maximum absolute $I A C F$ as IACC. An average of several $I A C C$ at different positions in the room is known to 
correlate with the ASW, especially in combination with bass strength. However, due to the high fundamental frequency of the played shakuhachi sound, no bass strength is present in the investigated instrument. $B Q I$ is defined as $1-I A C C$ so an increase in $B Q I$ results in an increased ASW. The $B Q I$ is known to show big fluctuations even at small position changes of the dummy head, which is not in accordance with the perceived ASW. Thus, again both the $B Q I$ itself and its fluctuations seem to have an impact on ASW. One adoption has to be made in this investigation to apply the $B Q I$ on pure direct sound from an exactly frontal incidence angle: $\tau$ needs to be 0 because no lateral sounds occur in the idealized complex point source model in a free field.

Parameters ILD, IPD and $B Q I$ are calculated for listeners at 128 listening angles at $1 \mathrm{~m}, 1.5 \mathrm{~m}$ and $3 \mathrm{~m}$ facing the instrument. This is done by comparing every third microphone signal which have a distance of about $0.15 \mathrm{~m}$ between each other at a distance of $1 \mathrm{~m}$ away from the source point which corresponds to the distance of two ears. Comparing every second microphone signal, propagated to a distance of $1.5 \mathrm{~m}$ from the source, again yields $0.15 \mathrm{~m}$ between each other. Comparing adjacent microphone signals equals a $0.15 \mathrm{~m}$ distance if propagating them $3 \mathrm{~m}$ away from the source, respectively. Thus, interaural differences can be calculated for 128 angles at three different distances from the source. Note, that the calculated "interaural" differences are in fact differences between propagated recording signals. Since the complex point source model assumes one source in the center and the listeners are assumed to face the source, the head-related transfer function HRTF is neglected. This is valid because a point source in the median plane does not create interaural differences at all and the path from source to ear is almost the straight connection line. Thus, a HRTF would only slightly filter the signal monaurally. Therefore, the terms "interaural" and "binaural" are used throughout this paper although no actual listener is involved.

\section{Analysis}

First, the spectra of the blown and overblown re are analyzed. One second of quasi-stationary sound is transformed into the frequency domain via DFT. To find the dominant partials, amplitudes of all 128 spectra without phase information are summed up. Including phase information would result in less distinct partials since amplitudes of out-of-phase-signals average out. The spectra of the blown and overblown note are plotted in Figure 2. In these, the first nine partials are clearly visible. These frequencies are considered for further analysis. It is conspicuous that partials of both notes deviate from the natural harmonic series. This probably results from the bore shape of the shakuhachi, see [8]. The overblown note is not actually the octave of the normally blown note but slightly higher. This is a typical phenomenon, probably caused by the fact that the shakuhachi was played by a novice flautist, cf. [9]. The fundamental frequency of the overblown note is much stronger compared to the overtones. Furthermore, as expected, the overblown note is much louder than the normally blown note which can be seen from the ratio of partials to noise.

Exemplarily, the second partial of the simply blown note $r e_{\text {blown }}(753 \mathrm{~Hz}, \varphi)$ and the first partial of the overblown
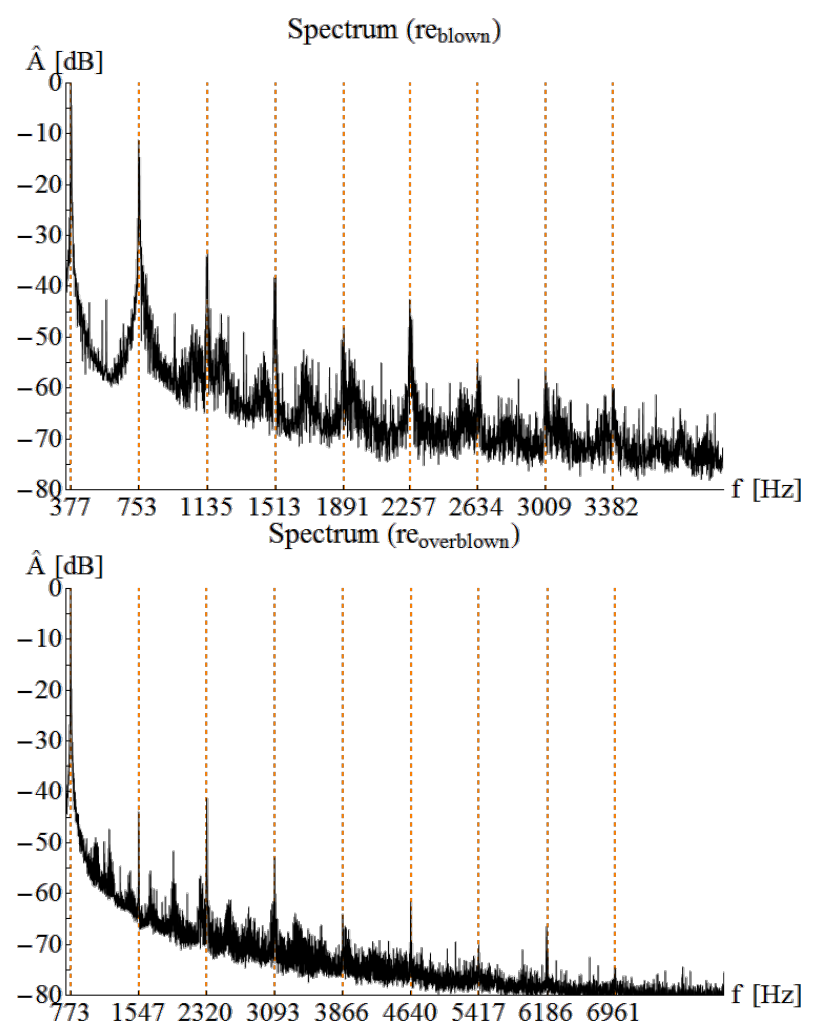

Figure 2: Spectra of blown (top) and overblown (bottom) note $r$. The first nine partials are considered for the analysis. They are marked by the dashed lines and their frequencies are given under the abscissa.

note $r e_{\text {overblown }}(773 \mathrm{~Hz}, \varphi)$ are plotted in this paper. The $I L D$ as calculated for a distance of $1 \mathrm{~m}, 1.5 \mathrm{~m}$ and $3 \mathrm{~m}$ are plotted in Figure 3. As expected, similar frequencies from both playing techniques with the same fingering create similar ILD patterns. They reach values of up to $24.21 \mathrm{~dB}$. Both ILD and their fluctuations decrease with increasing distance. They are higher for $r e_{\text {overblown }}$ than in case of $r e_{\text {blown. }}$ IPD show the same behavior as indicated in Figure 4: They are similar at similar frequencies, are bigger at higher frequencies, decrease with distance and are bigger in case of the overblown compared to the simple blown playing technique. They reach values up to 3.1 which is almost a phase inversion. The $B Q I$ as illustrated in Figure 5 behaves the same way.

$\oslash I L D, \oslash I P D, \oslash B Q I, R I L D, R I P D, R B Q I, \sigma I L D$, $\sigma I P D$ and $\sigma B Q I$ are summarized in Table 1 . They show the trends mentioned above. For higher frequencies the radiation patterns - and thus ILD, IPD and their fluctuations-appear to be more diverse. Two main statements can be made:

1. All considered quantities except $R I P D$ decrease with distance

2. All quantities except $R I P D$ are bigger for for $r e_{\text {overblown }}$ than for $r e_{\text {blown }}$

A simple listening test shall disclose whether listeners agree that the shakuhachi sounds smaller with increasing distance. Furthermore, it shall be shown if the blown and overblown note are perceived as having the same size or not. If they are, this would indicate that the magnitude of the considered quantities alone does not adequately describe perceived source size since the overblown note would sound bigger in that case. 


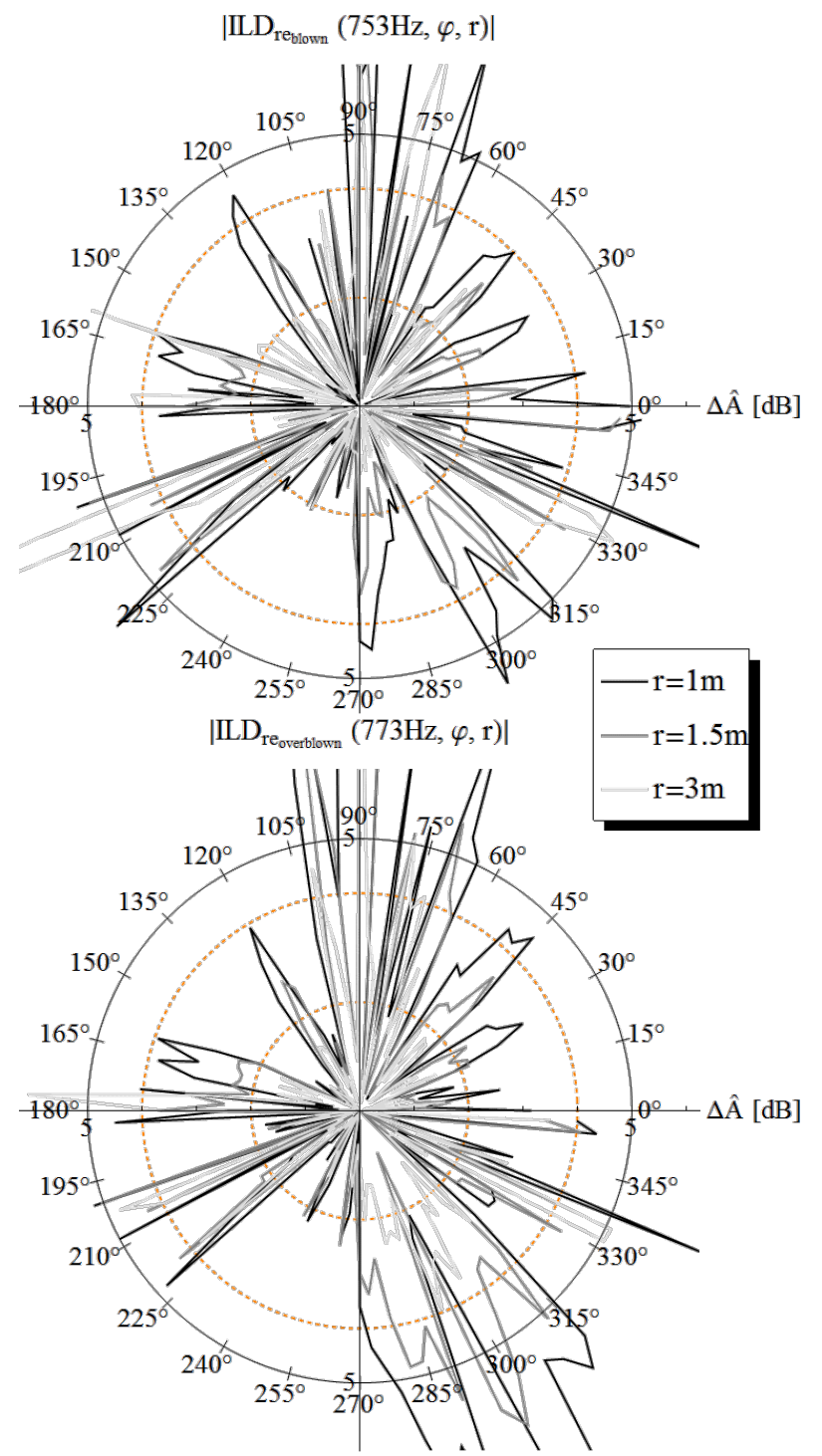

Figure 3: ILD of one frequency of a blown (top) and overblown (bottom) note at a distance of $1 \mathrm{~m}$ (black), $1.5 \mathrm{~m}$ (dark gray) and $3 \mathrm{~m}$ (light gray).

\subsection{Listening test}

Shakuhachi sounds are recorded in a free field room with a dummy head at an angle of $330^{\circ}$ at several distances. The recorded signals are then equalized with an inverse filter to eliminate the influence of the head and thus to externalize the recordings for playback via headphones. Several pairs of notes with different source distances and playing techniques, presented in randomized order, are compared by 14 subjects. They had to state whether the second sound of the pair appeared "closer" or not and "bigger" or not. For matters of comparison with our results from the microphone array measurement only $r e_{\text {blown }}$ and $r e_{\text {overblown }}$ at distances of $1 \mathrm{~m}$, $1.5 \mathrm{~m}$ and $3 \mathrm{~m}$ are considered. They are summarized in Table 2. In two of three cases with equal distances $r e_{\text {overblown }}$ is considered as big as $r e_{\text {blown }}$ but closer. In the other case $r e_{\text {blown }}$ sounds smaller but closer. $r e_{\text {overblown }}$ sounds both smaller and further away with increased distance. From this simple listening test three conclusions can be drawn:

1. $r e_{\text {overblown }}$ and $r e_{\text {blown }}$ sound equally big

2. The sources sound indeed smaller with increased

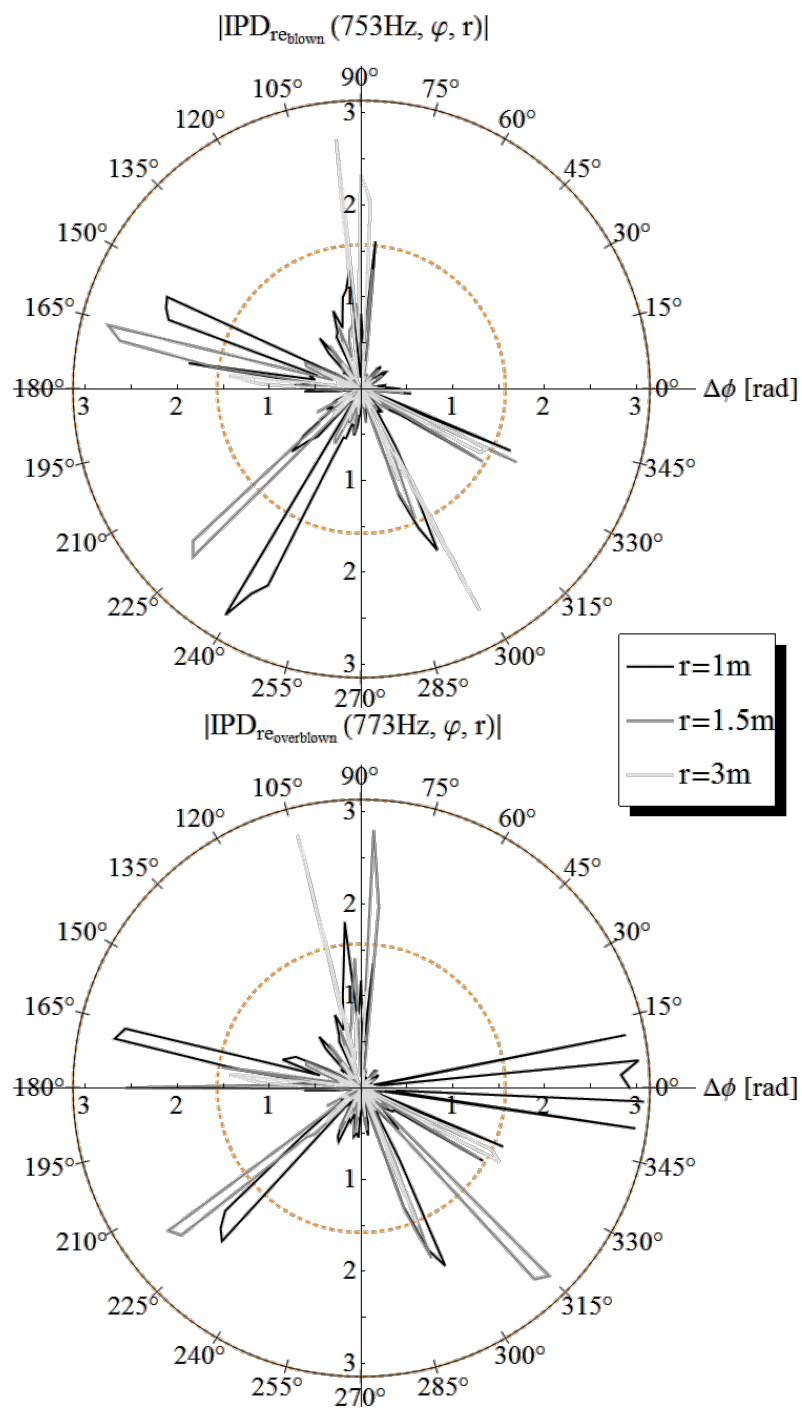

Figure 4: IPD of one frequency of a blown (top) and overblown (bottom) note at a distance of $1 \mathrm{~m}$ (black), $1.5 \mathrm{~m}$ (dark gray) and $3 \mathrm{~m}$ (light gray).

distance

3. The higher values for $r e_{\text {overblown }}$ seem to influence the perceived distance rather than the perceived source extent

Thus it is verified that the considered quantities agree with the perceived trend of decreasing source extent at increasing source distance but the magnitudes alone do not adequately describe the perceived source size. One important fact to mention is that although equalized for matters of externalization, many subjects reported an in-head localization.

\section{Conclusions and prospects}

The complex point source model has been introduced to measure, analyze and compare the radiation characteristics of musical instruments via a circular microphone array. With this method interaural level and phase differences as well as the binaural quality index and their fluctuations by means of range and standard deviation have been calculated for different listening angles and distances for two playing techniques of the shakuhachi. In general, the 


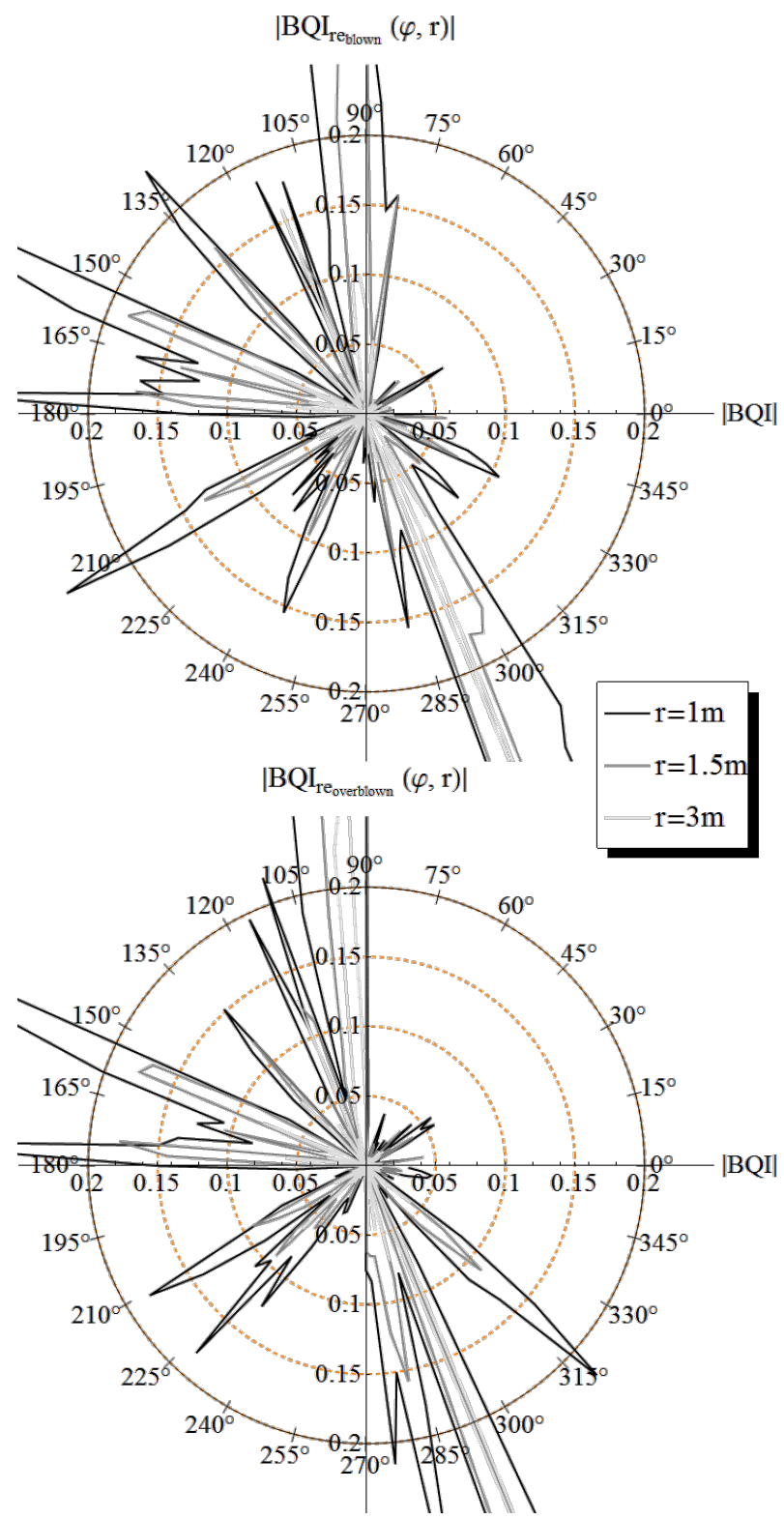

Figure 5: $B Q I$ of blown (top) and overblown (bottom) note at a distance of $1 \mathrm{~m}$ (black), 1.5m (dark gray) and 3m (light gray).

calculated quantities behave as expected: They decrease with increasing distance. This confirms the applicability of the method-at least for instruments as small and simple as the shakuhachi-and shows that ILD, R ILD, $\sigma I L D, I P D, R B Q I, \sigma I P D, B Q I$ and $\sigma B Q I$ seem to be appropriate measures to describe the effect that the perceived source extent decreases with distance. Only in case of the overblown note $R I P D$ does not decrease with distance. Furthermore, the overblown note creates typically higher interaural differences than the blown note, except $R I P D$. Certainly, one reason for the higher interaural differences in case of the overblown note is that it has a higher overall loudness and contains more energy in higher frequencies which tend to have a more complicated radiation characteristic. Still, results of the listening test do not show that the overblown shakuhachi note appear bigger than the normal blown note or that it increases with increasing distance. This indicates that the magnitude of interaural differences alone is not crucial for the perception of source extent. A ratio of $I L D$ and absolute level or a weighting of
Table 1: Mean interaural differences, ranges and standard deviations of 128 listening positions around the source at three different distances. In case of ILD and IPD it is furthermore the mean value of 9 frequencies.

\begin{tabular}{|c|c|c|c||c|c|c|}
\hline & \multicolumn{3}{|c||}{$r e_{\text {blown }}$} & \multicolumn{3}{c|}{$r e_{\text {overblown }}$} \\
\cline { 2 - 7 } & $1 \mathrm{~m}$ & $1.5 \mathrm{~m}$ & $3 \mathrm{~m}$ & $1 \mathrm{~m}$ & $1.5 \mathrm{~m}$ & $3 \mathrm{~m}$ \\
\hline$\oslash I L D$ & 4 & 3.28 & 2.37 & 4.71 & 3.99 & 3.14 \\
\hline$R I L D$ & 17.91 & 16.27 & 11.92 & 20.91 & 20.87 & 18 \\
\hline$\sigma I L D$ & 3.601 & 3.166 & 2.418 & 4.387 & 3.933 & 3.277 \\
\hline$\oslash I P D$ & 0.786 & 0.668 & 0.479 & 0.885 & 0.709 & 0.531 \\
\hline$R I P D$ & 0.967 & 0.913 & 0.472 & 0.53 & 0.515 & 0.571 \\
\hline$\sigma I P D$ & 0.694 & 0.675 & 0.631 & 0.82 & 0.749 & 0.67 \\
\hline$\oslash B Q I$ & 0.022 & 0.013 & 0.007 & 0.104 & 0.062 & 0.026 \\
\hline$R B Q I$ & 0.09 & 0.06 & 0.04 & 0.12 & 0.08 & 0.02 \\
\hline$\sigma B Q I$ & 0.018 & 0.011 & 0.007 & 0.17 & 0.141 & 0.093 \\
\hline
\end{tabular}

Table 2: Results from the listening test with 14 subjects. Cases in which perceived size and distance differ by more than 2 are gray.

\begin{tabular}{|l|c|c|}
\hline & Bigger & Closer \\
\hline $1 \mathrm{~m}$ blown & 7 & 5 \\
$1 \mathrm{~m}$ overblown & 7 & 9 \\
\hline $1.5 \mathrm{~m}$ blown & 10 & 4 \\
$1.5 \mathrm{~m}$ overblown & 3 & 9 \\
\hline $3 \mathrm{~m}$ blown & 7 & 4 \\
$3 \mathrm{~m}$ overblown & 7 & 9 \\
\hline $1 \mathrm{~m}$ overblown & 12 & 13 \\
$1.5 \mathrm{~m}$ overblown & 2 & 0 \\
\hline
\end{tabular}

interaural differences by a frequency-dependent factor may be a more suitable quantity to describe perceived absolute source extents. Measuring bigger musical instruments with more complicated geometries using the proposed method will show in which limits instruments can still be considered as complex point sources. For quantitative analysis and comparisons of interaural measures of different musical instruments, more instruments need to be measured in the described way. Listening tests with more subjects, more instruments and a higher measurement scale might reveal correlations between physical quantities and perceived source extent as achieved in the field of subjective room acoustics. A listening test setup which allows for head movements may yield more reliable results concerning the influence of fluctuations of interaural measures.

\section{References}

[1] J. Meyer, Acoustics and the Performance of Music, 5th ed., Springer, New York (2009) 
[2] J. Pätynen, T. Lokki, "Directivities of Symphony Orchestra Instruments", Acta Acustica United With Acustica 96, 138-167 (2010)

[3] F. Zotter et al., "Capturing the Radiation Characteristics of the Bonang Barung", 3rd Congress of the Alps Adria Acoustics Association (2007)

[4] R. Bader, et al., "Measurements of Drums and Flutes", R. Bader (ed.), Musical Acoustics, Neurocognition and Psychology of Music, 15-55, Peter Lang, Frankfurt a.M. (2009)

[5] M. B. S. Magalhăes, R. A. Tenenbaum, "Sound Sources Reconstruction Techniques: A Review of Their Evolution and New Trends", Acta Acustica United with Acustica 90, 199-220 (2004)

[6] D. Griesinger, "Objective Measures of Spaciousness and Envelopment", AES 116th International Conference on Spatial Sound Reproduction (1999)

[7] T. Okano, L. L. Beranek, T. Hidaka, "Relations among interaural cross-correlation coefficient $\left(I A C C_{E}\right)$, lateral fraction $\left(L F_{E}\right)$, and apparent source width (ASW) in concert halls", J. Acoust. Soc. Am. 104(1), 255-265 (1998)

[8] Y. Ando, "On Bore Shape of a Shakuhachi and its Resonance Characteristics”, JCAS 47, 21-25 (1987)

[9] P. de la Cuadra, B. Fabre, C. Chafe, "Analysis of Flute Control Parameters: A Comparison Between a Novice and an Experienced Flautist", Acta Acustica United with Acustica 94, 740-749 (2008) 